\title{
Reconstruction of the geometry of volcanic vents by trajectory tracking of fast ejecta - the case of the Eyjafjallajökull 2010 eruption (Iceland)
}

\author{
Tobias Dürig $^{1^{*}}$, Magnus T Gudmundsson ${ }^{1}$ and Pierfrancesco Dellino ${ }^{2}$
}

\begin{abstract}
Two methods are introduced to estimate the depth of origin of ejecta trajectories (depth to magma level in conduit) and the diameter of a conduit in an erupting crater, using analysis of videos from the Eyjafjallajökull 2010 eruption to evaluate their applicability. Both methods rely on the identification of straight, initial trajectories of fast ejecta, observed near the crater rims before they are appreciably bent by air drag and gravity. In the first method, through tracking these straight trajectories and identifying a cut-off angle, the inner diameter and the depth level of the vent can be constrained. In the second method, the intersection point of straight trajectories from individual pulses is used to determine the maximum possible depth from which the tracked ejecta originated and the width of the region from which the pulses emanated. The two methods give nearly identical results on the depth to magma level in the crater of Eyjafjallajökull on 8 to 10 May of $51 \pm 7 \mathrm{~m}$. The inner vent diameter, at the level of origin of the pulses and ejecta, is found to have been 8 to $15 \mathrm{~m}$. These methods open up the possibility to feed (near) real-time monitoring systems with otherwise inaccessible information about vent geometry during an ongoing eruption and help defining important eruption source parameters.
\end{abstract}

Keywords: Explosive volcanism; Ejecta trajectory tracking; Vent geometry; Near-field monitoring; Pulsating explosive eruptions; Eruption source parameters; Video analysis

\section{Findings}

\section{Introduction}

The monitoring of the mass flux of an explosive eruption whether if using ash plume models, video cameras, or infrasound sensors - requires constraints on the vent-crater geometry. A potential way to obtain such constraints is through careful measurements of paths and velocities of ejecta emitted from a volcanic vent. Tracking of pyroclastic particles by video analysis has been shown to be a tool that gives valuable insights into crater processes (e.g., Hort et al. 2003) as well as on the pressure (e.g., Taddeucci et al. 2012; Gaudin et al. 2014) and energy balances (e.g., Maeno et al. 2013) of explosive eruptions. To date, such studies have been based on the analysis of ballistic trajectories (e.g., Mastin 2008).

\footnotetext{
* Correspondence: tobi@hi.is

${ }^{1}$ Nordic Volcanological Center, Institute of Earth Sciences, University of Iceland, Sturlugata 7, 107, Reykjavík, Iceland

Full list of author information is available at the end of the article
}

We present here complementary methods for the analysis of the initial (yet unbent) flight paths of the ejecta. From this analysis, information is obtained about the vent-crater geometry, which helps constraining the characteristic of the particle source, i.e., its depth level $z$ where individual ejecta trajectories are decoupled, and the inner vent radius $r$. While the first parameter is of importance for volumetric analyses of ash pulses in the initial gas thrust regime, allowing an estimate of the total mass flux (see, e.g., Dürig et al. 2014), the vent radius $r$ is an important input parameter for any plume model (e.g., Sparks et al. 1997; Suzuki and Koyaguchi 2013; Degruyter and Bonadonna 2012; Woodhouse et al. 2013). The present analysis allows also a more realistic definition of the uppermost part of the volcanic conduitcrater system (see, e.g., synthetic aperture radar (SAR) image of Eyjafjallajökull on 11 May in Figure 1) by treating it as funnel-shaped - rather than having the shape of a cylinder. 


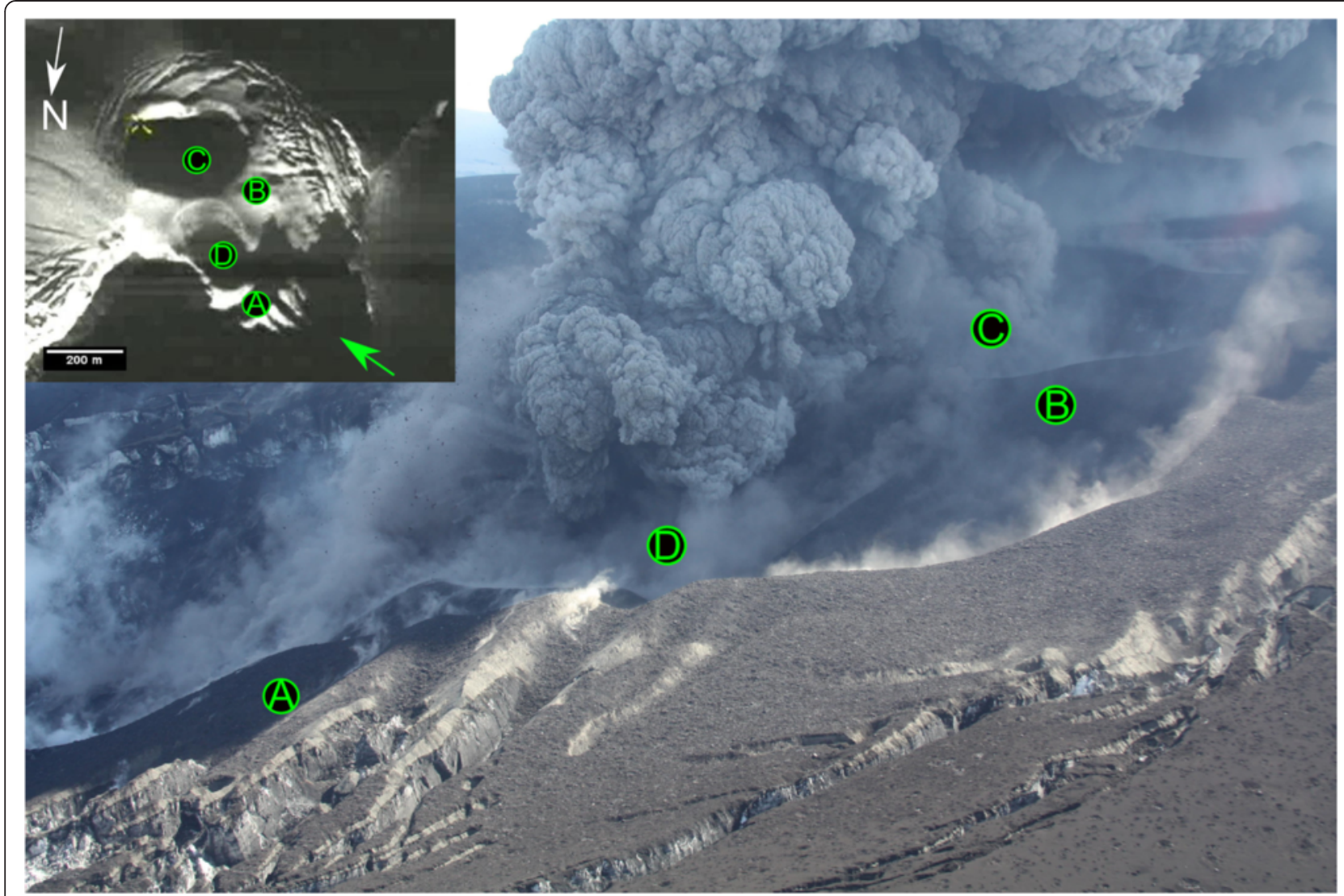

Figure 1 Eyjafjallajökull crater situation during 8 to 10 May 2010. Specific features (labelled 'A' to 'D') visible in an aerial image taken at 8 May can also be identified in an airborne SAR image taken 3 days later (inlet), suggesting that the crater geometry has not significantly changed during this period. The green arrow indicates the angle of view of the video camera which was placed at 850-m distance from the center (' $\mathrm{D}$ ') of the active vent. Based on such images and radar maps, it was possible to estimate the outer vent radius $R$ and the angle of repose $\beta$. Image courtesy of Thórdís Högnadóttir.

Our new methods are applied to video sequences shot during the 2010 eruption of Iceland's Eyjafjallajökull (excerpts from these videos are found in Rietze (2010)). The eruption, which attracted the attention of hundreds of thousands of stranded flight passengers in Europe, consisted of multiple closely timed pulses (e.g., Langmann et al. 2011; Dellino et al. 2012; Gudmundsson et al. 2012) that merged at a higher altitude in a continuous eruption column. We perform an analysis of images from videos of 502 pulses that were recorded during the period 8 to 10 May (see also Figure 1). Both the position of the video camera (at the summit caldera rim, $850 \mathrm{~m}$ to the northwest of the vent) and good visibility conditions facilitated near-field analyses of the early expansion phase as well as the identification of individual ejecta trajectories.

\section{Methods}

Two approaches were used to reconstruct the depth and width of the pulse source level, denoted 'cut-off angle method' (CAM; see Figure 2a) and 'trajectory intersection approach' (TIA; see Figure 2b). For both methods, only the lower (near vent) segments that remain straight (unbent) are analyzed. These are considered to be the early flight paths of high-velocity ejecta for which the work of gravity, wind speed, and air drag are (yet) so small relative to their momentum (see Figure 2c) that they do not significantly affect the trajectory paths.

Figure 2a illustrates the basic idea of the CAM: given a vent with an inner radius $r$, an angle of repose of the funnel-shaped inner crater $\beta$, and an outer vent radius $R$, particles ejected with a straight trajectory originating from a depth $z$ cannot show up with an ejecting angle lower than $\alpha$. Trigonometric considerations lead to the two relations:

$$
\tan \beta=\frac{z}{R-r}
$$

and

$$
\tan \alpha=\frac{z}{R+r}
$$

Transforming (1) results in: 

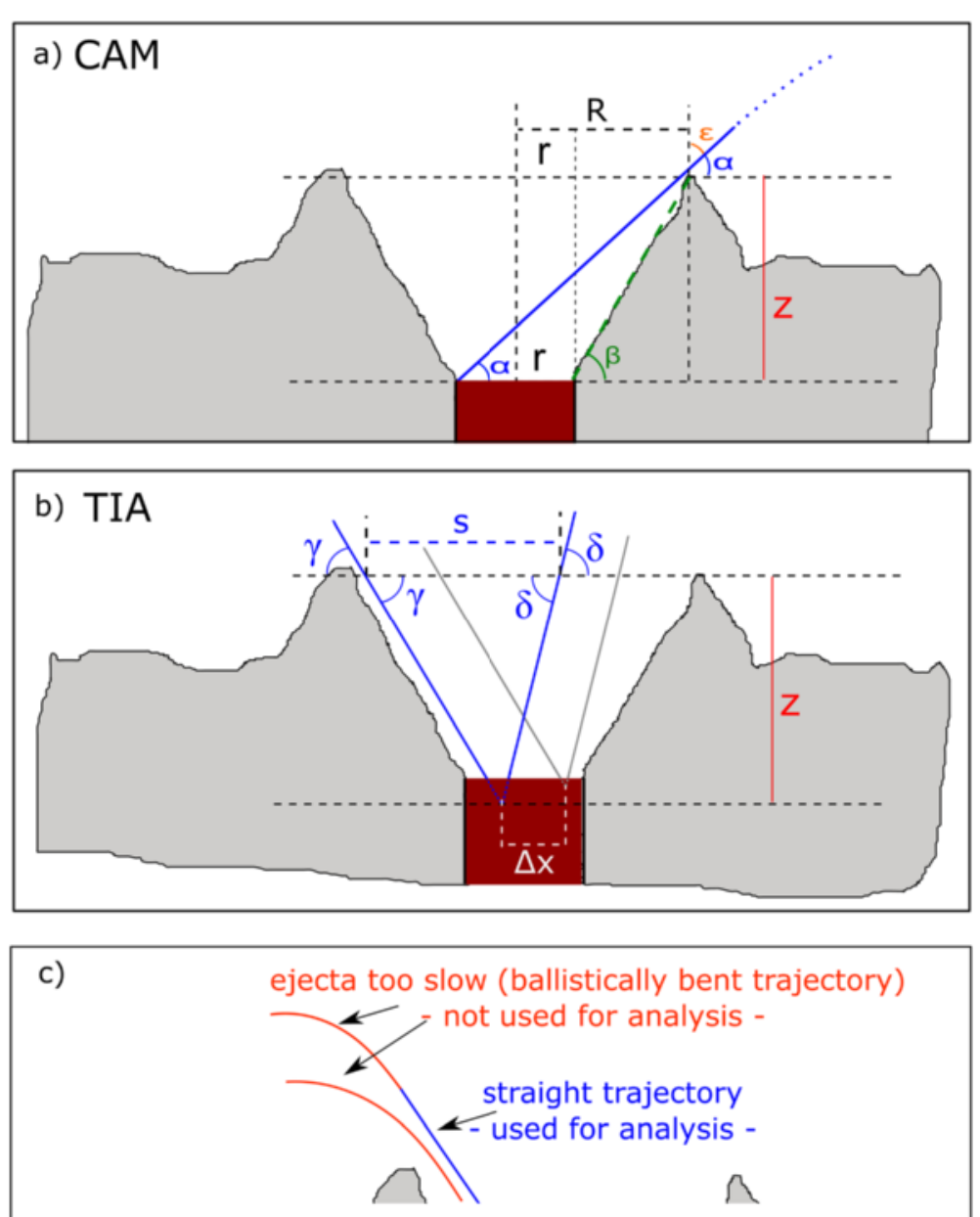

Figure 2 Methods used to reconstruct the vent geometry via trajectory tracking. (a) 'Cut-off angle method' (CAM): by analyzing all straight (unbent) trajectories of high-speed particles, the cut-off angle, i.e., the maximum of all possible ejection angles $\varepsilon$, can be determined and used to constrain the inner vent radius $r$ and the depth $z$ of their origin. (b) 'Trajectory intersection approach' (TIA): by measuring the exit angles $\gamma$ and $\delta$ of two straight trajectories within an individual pulse and their horizontal distance $s$ at the exit of the vent, it is possible to determine the depth of the intersection point $z$. A comparison between the different pulses reveals possible vertical $(\Delta z)$ and horizontal variations $(\Delta x)$ of the intersection points. (c) In contrast to traditional ballistic trajectory approaches, both methods presented use only straight trajectories, i.e., flight paths of high-speed particles (indicated in blue). Bent trajectories (marked in red) are neglected.

$$
r=R-\frac{z}{\tan \beta}
$$

Inserting (3) into (2) and solving the equation for $z$ gives the expression:

$$
z=2 \cdot R \cdot \tan \alpha /\left(1+\frac{\tan \alpha}{\tan \beta}\right)
$$

After determining $R, \beta$, and the cut-off angle of all ejecta for each pulsation, it is hence possible to estimate $z$, the depth level of origin of particles with individual trajectories, by applying (4) and the inner vent radius $r$ by (3).

In order to determine the minimum ejection angle $\alpha$, image stacking was applied. Figure 3a shows an example of a pulse, recorded at GMT 21.55 h of 8 May 2010. For the analysis of this pulse, a sequence of 20 frames from a 30-fps video was stacked, and the maximum brightness values were calculated pixel by pixel by using the software Image J (Schneider et al. 2012). This operation allowed the trajectories of ejected particles to be visible against the dark background, since the pulse occurred during the evening. However, this procedure turned out to be less effective for videos shot under daylight conditions.

Hence, a more complex method was used to process video sequences, which makes trajectories of individual particles visible under all background conditions: first, the videos were cut into sequences of 60 stacked frames (i.e., 2 s). Mathematically, these stacks can be written as a suite of matrices. Then, for each frame, the pixel-bypixel differences of the brightness values relative to the previous frame were calculated: 


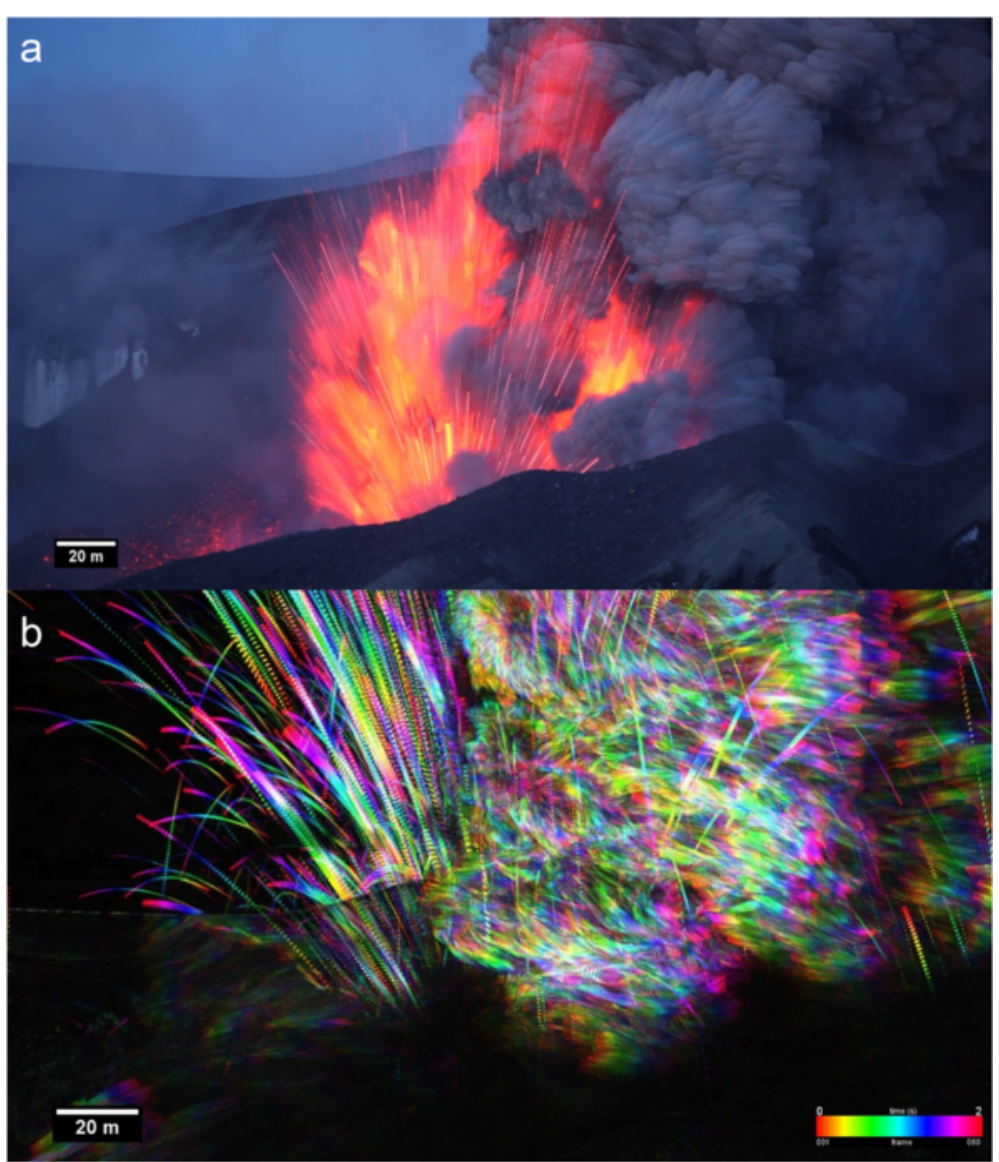

Figure 3 Image stacking was applied to identify and measure the trajectories of clasts. (a) The upper frame gives an impression of the number of particles ejected during a pulse (20 frames were stacked; this is essentially the picture that would have been created by a camera with an exposure time of $2 / 3 \mathrm{~s}$ ). As this way of visualization only works at night and high contrast to a dark background, another standardized method was applied to track the trajectories: (b) 60 frames (i.e., $2 \mathrm{~s}$ of video footage) were stacked; the difference to each frame was calculated pixel by pixel and colored according to the respective frame. The image shows bent and straight trajectories, but only the latter were used for analysis. The visibility decreases on the right (downwind) side and is particularly low in the first quadrant $\left(0^{\circ}\right.$ to $\left.90^{\circ}\right)$, as this sector is covered by ash clouds.

$$
\left(R_{i}\right)_{i=1, \ldots, 60}=\left\{\begin{array}{cc}
X_{i}-X_{i+1} & \text { for } X_{i}-X_{i+1}>0 \\
0 & \text { for } X_{i}-X_{i+1} \leq 0
\end{array}\right.
$$

In the resulting images, pixels with a high difference of brightness are rendered bright, while pixels with no or with low brightness difference are displayed in black or dark grey colors. Finally, the temporal change is colored by assigning a specific color to each frame. This is done by using the plugin 'Temporal-Color Coder' in ImageJ which provides as a last step a merged image that shows pixel by pixel the frame number in which the maximum luminosity of the stacked frames is reached (Miura 2008). An explanatory example of such a merged picture is shown in Figure 3b.

All trajectories were compared with manually overlaid straight lines of a length equivalent to at least $40 \mathrm{~m}$. A trajectory was defined as 'bent' and ruled out for further analysis if a discrepancy between the flight path and the straight line corresponding to a deviation of more than 1 pixel (equivalent to approximately $0.2 \mathrm{~m}$ ) was found along the 40-m line.

In order to check the consistency of the results obtained by the CAM approach, an alternative method, the TIA, was used, which is depicted by Figure $2 b$. The idea is to find the point of origin (trajectories intersection point) of particles ejected within individual pulses. By measuring the angles $\gamma$ and $\delta$ of two straight trajectories and their distance $s$ at the exit of the vent, the depth of the intersection point $z$ can be calculated with:

$$
z=s \cdot \tan \delta /\left(1+\frac{\tan \delta}{\tan \gamma}\right)
$$

Besides presuming a point source, the TIA does not need any further assumptions neither about the source of the ejecta nor about the source parameters (such as 
the width of an individual bursting bubble). In Figure $2 b$, a second pulse is indicated in grey color. The points of intersection show variances in both vertical and horizontal directions.

Since the uncertainty of the $z$ values resulting from Equation (6) increases with steeper angles, the outermost straight trajectories are preferred rather than the arbitrary straight flight paths.

\section{Results}

The video sequences analyzed refer to a phase of the volcanic activity when the eruption rate had dropped significantly from the maximum output of the second explosive phase, which occurred on 5 to 6 May (Gudmundsson et al. 2012). As a consequence, a smaller vent had started to develop within the larger vent that was active during the period of maximum activity. Our observations apply to this inner vent (marked with 'D' in Figure 1).

For both methods, CAM and TIA, it is assumed that the vent axis is vertical and that there is symmetry in the direction of ejecta relative to the vertical axis of the vent.

\section{Trajectory angles}

In total, the trajectory angles of 2,479 individual particles were determined $(1,160,140$, and 1,179 trajectories for 8, 9, and 10 May, respectively). Figure 4, in which a counterclockwise notation for ejection angles $\varepsilon$ towards the vertical is adopted, shows the resulting histogram. The majority of trajectory angles lies between $62^{\circ}$ and (roughly) $-38^{\circ}$. Only in a few cases flatter angles were observed. Notably, there was no variance in these angles during the 3 days of recording (cf. Figure 4, particularly for 8 and 10 May, for which a similar amount of data i.e., footage - was available), indicating a stable geometry of the vent system over that time period. The lack of significant differences between aerial photos from 8 May and airborne SAR images from 11 May supports this hypothesis (see Figure 1).

Due to the wind always coming from the left side (approximately from the north, for illustration see also Figure 4), the angles of the first quadrant (leeward right sector) were partly covered by contemporaneous ash clouds; hence, the trajectories of the ejecta could not be observed to the same extent as those ejected within the second observation quadrant (left sector). Also a part of

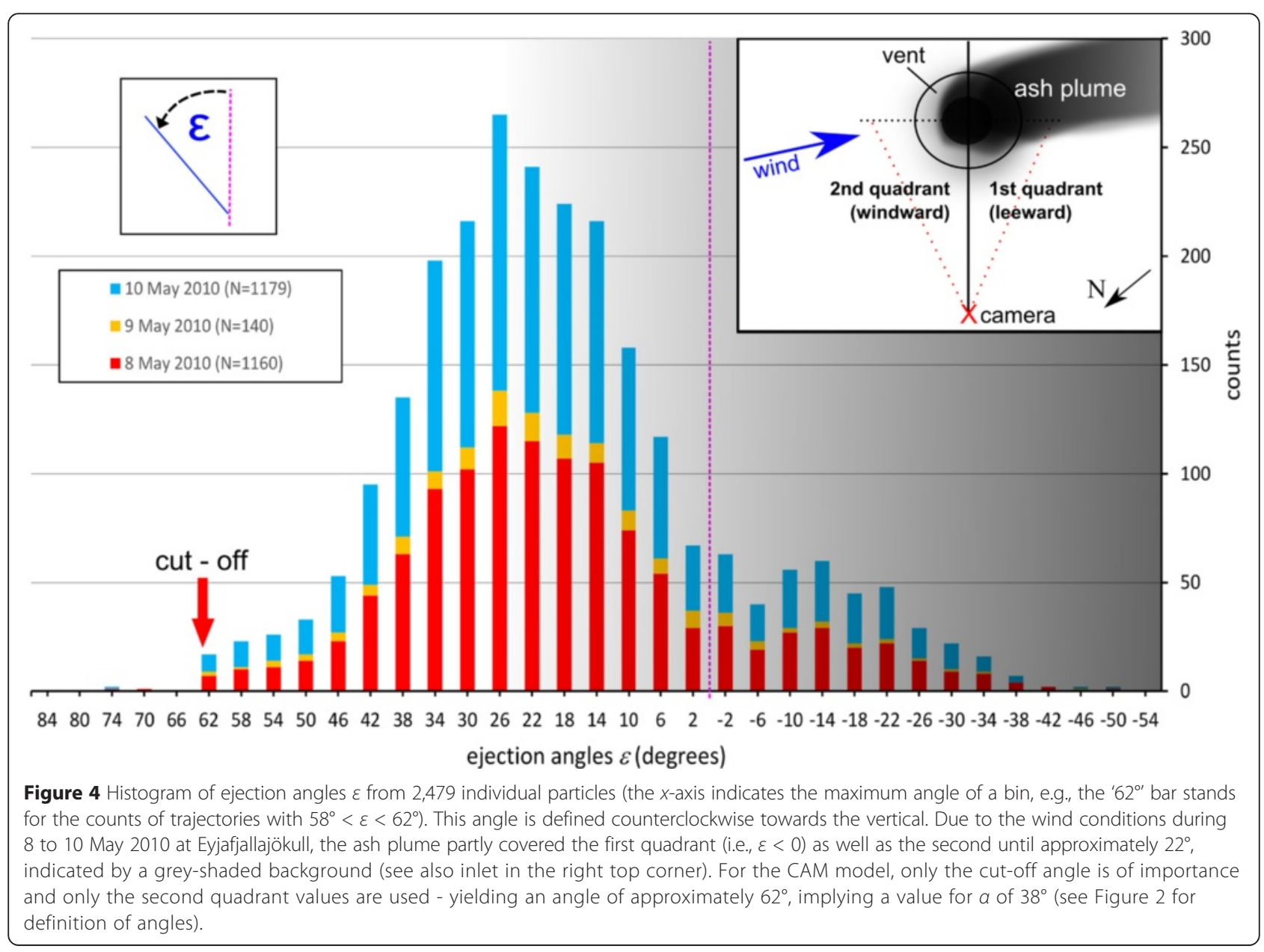


the second quadrant was affected: the number of ejecta, which would - presuming a vertical conduit - be expected to be maximum at $0^{\circ}$ (see vertical line in Figure 4), shows instead a maximum at $26^{\circ}$. This can be explained by the increasing lack of visibility, indicated by the gradual grey haze in Figure 4. As a consequence, we decided to focus on the statistics of the (apparently more significant) windward second quadrant, particularly for the ejection angles $>26^{\circ}$, in order to find the flattest angle.

\section{Occurrence of particle-particle collisions}

A possible source of error of our method can be the deflection from the original trajectory due to clast-clast collisions inside the conduit. Such errors would affect the data significantly only if all of the following conditions are matched:

1. The collision occurs close to the vent exit.

2. One or more of the colliding particles (or their parents in case of disintegration) are deflected at high velocities resulting in straight post-collision trajectories.

3. The resulting deflection angles are lower than the 'real' cut-off angle from the perspective of the camera.

Our observations suggest that the likelihood that all three conditions are matched is very low: within the 35 min of analyzed videos, a total of 97 clast-clast collisions was counted outside the vent. This is a very small ratio, considering the fact that during that period thousands of ejecta were observed (including the 2,479 particles that were attributed with straight flight paths). The collisions that were observed always resulted from a downwardfalling particle colliding with upward moving (just ejected) clasts. Most of the observed cases were hits under very steep angles. In the rarer cases in which the ejecta of flatter angles were hit by downward-falling particles, the impact was characterized by the formation of a dust cloud, followed by a spreading cloud of quickly disintegrating particles that travelled along typical ballistic trajectories. Only in one case were conditions 2 (straight post-collision trajectories) and 3 (resulting angle larger than $62^{\circ}$ to the vertical) matched.

The complex matter of particle-particle collision is an ongoing topic of current volcanological research (see, e.g., Cagnoli and Manga 2003, Tsunematsu et al. 2014). Although observations outside the vent certainly cannot be seen as a 'proof' for inside clast-clast collisions, our data, based on 2,479 tracked particles, might serve at least as an indication of the low probability of occurrence of 'ricochets' that match the three conditions mentioned above.
Figure 4 shows an evident cut-off for $\varepsilon$ larger than $62^{\circ}$ which corresponds to a cut-off angle $\alpha$ of $38^{\circ}$. Only three (of the 2,479) trajectories showed flatter angles. According to the considerations above, these outliers were assumed to be the results from clast-clast collisions and were therefore discarded from the dataset.

\section{Depth to magma level and maximum vent diameter estimate, CAM approach}

It has to be considered that maximum angles of straight trajectories should be affected only by the vent profile in the plain perpendicular to the line of view. By means of photos taken from aircraft on 8 and 11 May as well as airborne SAR images from 11 May, the outer radius $R$ of this inner vent of Eyjafjallajökull on 8 to 10 May (perpendicular to the axis of view) was determined as $65 \pm 2$ $\mathrm{m}$ and the angle of repose $\beta$ was $38^{\circ} \pm 5^{\circ}$. By inserting, in Equation (4), these values of $R$ and $\beta$, and $38^{\circ} \pm 1^{\circ}$ for $\alpha$, the resulting depth level $z$ is $51 \pm 7 \mathrm{~m}$. The uncertainty of $z$ was estimated by varying the three input parameters within their margins of error. When the same variational approach is applied to Equation (3), the maximum value of the inner vent diameter, $2 r$, is found to be $15 \mathrm{~m}$; a minimum value cannot be estimated with this method.

\section{Depth to magma level and minimum vent diameter estimate, TIA method}

The second, independent TIA method, was applied to 42 pulses. The exit angles $\delta$ and $\gamma$ were determined and used as input parameters for Equation (6). The resulting values for $z$ are plotted in Figure 5. The uncertainties of TIA-derived $z$ values were individually quantified by varying all input parameters of Equation (6) within their margins of error, i.e., $\pm 1^{\circ}$ for $\delta, \pm 1^{\circ}$ for $\gamma$, and $\pm 0.5 \mathrm{~m}$ for $s$. The resulting $z$ values range between $42 \pm 2 \mathrm{~m}$ and $61 \pm 3$ $\mathrm{m}$ and average $51 \mathrm{~m}$. Hence, the results derived by TIA coincide with the range of $z$ provided by the CAM, i.e., $51 \pm 7$ $\mathrm{m}$ (indicated by dashed green lines within Figure 5). The intersection points of the 42 pulses analyzed for the TIA method define a region of width $\Delta x$, which may correspond to the width of the conduit. The value of $\Delta x$ for the pulses analyzed was found to be $8 \mathrm{~m}$.

\section{Discussion}

Both methods provide consistent results, although the CAM is expected to be more robust than the TIA, as the first method uses cumulative pulse trajectories and is therefore based on a much larger, statistically more significant data set. Our findings indicate that when a sufficiently large number of pulses is used, the TIA provide results on depth to source level of a quality comparable to those derived by the CAM. This conclusion is of 


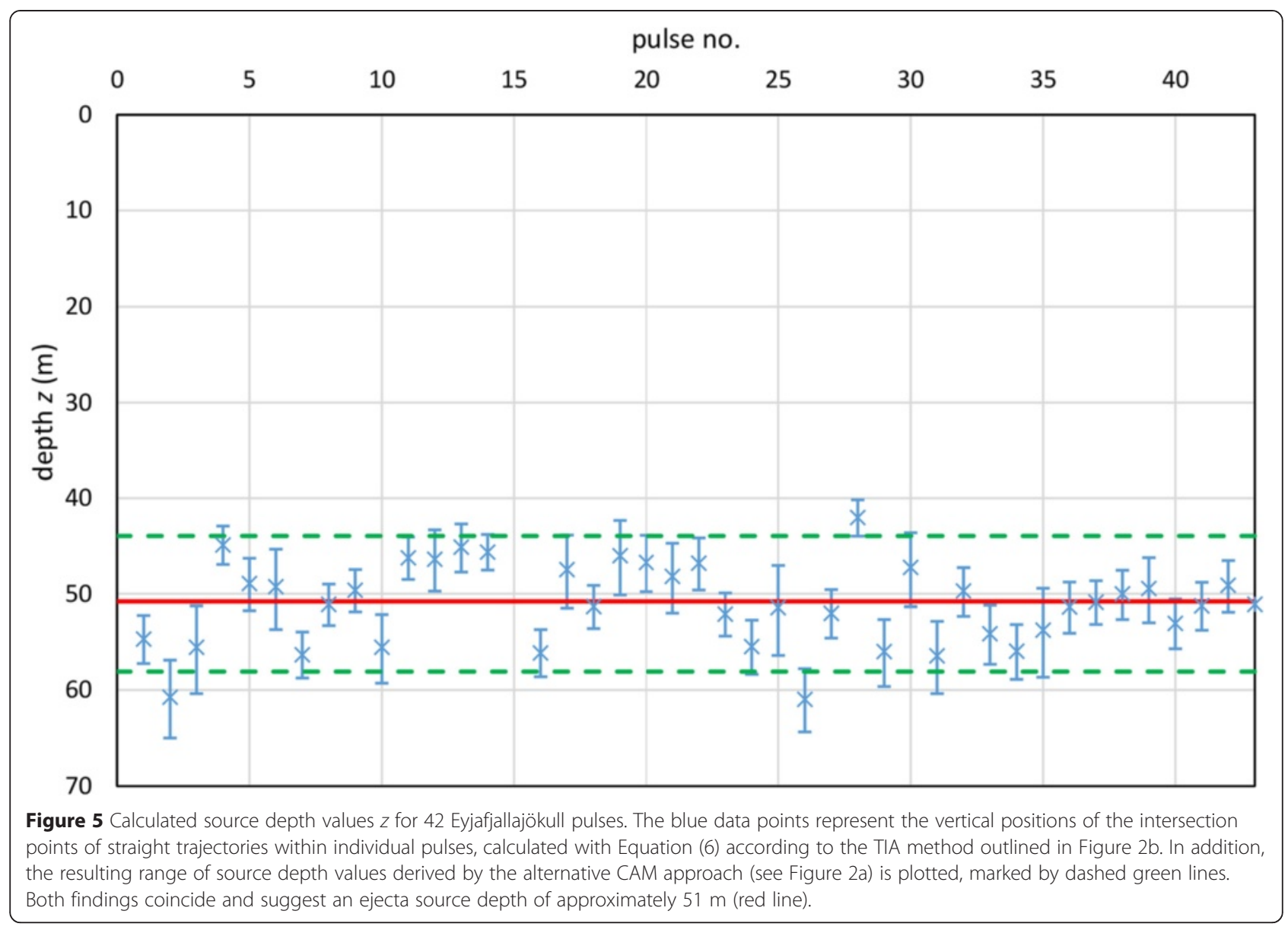

interest, as the TIA is easier to apply and needs less knowledge of parameters than the CAM.

The depth of the source level $z$ is, according to our consideration, the depth from which the ejecta trajectories separate from each other, which means it can be considered as the depth from which the eruption column starts to expand. Thus, the empirically defined parameter $z$ does not necessarily coincide with the level of magma fragmentation; that level may be slightly lower in the conduit but will not be considered further here.

The TIA method could in principle also be used for straight trajectories confined to only one side of the symmetry axis of the pulse. However, the smaller difference between the steepest and the flattest angle would lead to a higher uncertainty; therefore, we only analyzed pulses generating straight trajectories visible on both sides. Pulses within the right-hand side (leeward) sector that were partly (or completely) 'hidden' by ash were hence not considered with the TIA. Although it relies to some extent on the assumption of a symmetrical vent, the CAM suffers less from this limitation, as for that method only the cut-off angle of the cumulative straight trajectories of one side was analyzed. Against this background, the results of the horizontal deviation $\Delta x$ found by TIA can be seen as a minimum estimate for the inner vent diameter $2 r$, while CAM in combination with the variation of the input parameters provides a maximum value. Thus, we conclude that the diameter of the inner vent at source level ranged between 8 and $15 \mathrm{~m}$.

The horizontal variation of intersection points of straight trajectories from individual pulses, together with the fact that the depth of intersection points lies within the range of $z$ derived by CAM, might also serve as an indication for a typical pulse width: a pulse which has an initial width of $2 r$ should be characterized by intersection points that are up to approximately $10 \mathrm{~m}$ deeper than the $z$ value provided by CAM. The fact that there is no significant difference between the result of CAM and the depth values of intersection points suggests that the pulses analyzed were punctuating jets with a comparatively small initial width, rather than bursts with a width of $2 r$.

It is important to consider that both CAM and TIA provide maximum estimates for $z$ : the TIA provides the depth of intersection points, which have to be considered as maximum possible depth of the source.

The CAM assumes that the flattest angle visible is that of the ejecta which originate from the margin of the 
vent. In the case of a 'blind area' at the source level from which no clast is ejected, the observed cut-off angle would be too steep, leading to an overestimate of $z$. However, error analysis reveals that due to the fact that $z \gg r$, the sensitivity of the cut-off angle towards this effect is low. For demonstration, let us assume $z$ to be $50 \mathrm{~m}$ and the (real) inner vent diameter $2 r$ to be $10 \mathrm{~m}$. A 'blind area' of 1-m length on each side (i.e., $20 \%$ of the diameter) would lead to an error for $\alpha$ of approximately $1^{\circ}$, meaning that $\alpha$ would still be within the above assumed uncertainty.

\section{Conclusions and outlook}

The methods presented in this paper are simple tools that allow constraining the geometrical parameters of the vent during an eruption, provided that a video of adequate quality can be obtained. They can be easily automated by applying clast tracking software, like that of Gaudin et al. (2014). This means that if an eruption is monitored by a camera and videos are processed in near real-time, the (inner) vent radius $r$ as well as the depth level $z$ of emitted ash pulses could be assessed. The importance of these input parameters for any models of mass flux, including plume models, has been mentioned above. In addition, it is worth considering that they are also essential to monitoring techniques such as infrasound sensing. Infrasound interpretation depends on a robust estimate of the effective vent radius (Ripepe et al. 2013). Hence, the methods presented here would be a useful complement to any real-time multi-parameter system that constrains the mass eruption rate, which is the main source parameter influencing ash plume formation and higher-up atmospheric circulation.

\section{Competing interests}

The authors declare that they have no competing interests.

\section{Authors' contributions}

TD performed the analyses and led the development of the models and ideas presented in collaboration with MTG and PD. All authors contributed to the ideas and models presented in this paper and took part in writing the paper. All authors read and approved the final manuscript.

\begin{abstract}
Acknowledgements
The authors would like to express their gratitude to Martin Rietze for kindly providing his footage and encourage the reader to visit his webpage www.mrietze.com. We thank also Kota Miura (EMBL, Heidelberg, Germany) for the Temporal-Color Code ImageJ plugin. The photo obtained on 8 May was acquired by Thordís Högnadóttir, Univ. of Iceland. The work of TD is founded by the EU FP7 supersite project FutureVolc. Finally, we would like to thank two anonymous reviewers for their most useful comments and suggestions which helped us to improve the quality of our paper.
\end{abstract}

\section{Author details}

${ }^{1}$ Nordic Volcanological Center, Institute of Earth Sciences, University of Iceland, Sturlugata 7, 107, Reykjavík, Iceland. ${ }^{2}$ Dipartimento di Scienze della Terra e Geoambientali, Via Orabona, 4, Università di Bari, 70125 Bari, Italy.

\section{References}

Cagnoli B, Manga M (2003) Pumice-pumice collisions and the effect of the impact angle. Geophys Res Lett 30:1636, doi:10.1029/2003GL017421

Degruyter W, Bonadonna C (2012) Improving on mass flow rate estimates of volcanic eruptions. Geophys Res Lett 39, L16308, doi:10.1029/2012GL052566

Dellino P, Gudmundsson MT, Larsen G, Mele D, Stevenson JA, Thordarson T, Zimanowski B (2012) Ash from the Eyjafjallajökull eruption (Iceland): fragmentation processes and aerodynamic behaviour. J Geophys Res 117:B00C04. doi: 10.1029/2011JB008726

Dürig T, Gudmundsson MT, Karmann S, Zimanowski B, Dellino P, Rietze M, Büttner R (2014) Dynamics of eruptive pulses - a case study of the second explosive phase of the 2010 Eyjafjallajökull eruption (Iceland). In: Geophysical Research Abstracts 16:EGU2014-4083-1. EGU General Assembly, Vienna

Gaudin D, Taddeucci J, Scarlato P, Moroni M, Freda C, Gaeta M, Palladino DM (2014) Pyroclast Tracking Velocimetry illuminates bomb ejection and explosion dynamics at Stromboli (Italy) and Yasur (Vanuatu) volcanoes. J Geophys Res Solid Earth 119:1-14

Gudmundsson MT, Thordarson T, Höskuldsson A, Larsen G, Björnsson H, Prata FJ, Oddsson B, Magnússon E, Högnadóttir T, Petersen GN, Hayward CL, Stevenson JA, Jónsdóttir I (2012) Ash generation and distribution from the April-May 2010 eruption of Eyjafjallajökull, Iceland. Sci Rep 2:572, doi:10.1038/srep00572

Hort M, Seyfried R, Vöge M (2003) Radar Doppler velocimetry of volcanic eruptions: theoretical considerations and quantitative documentation of changes in eruptive behaviour at Stromboli volcano, Italy. Geophys J Int 154:515-532

Langmann B, Folch A, Hensch M, Matthias V (2011) Volcanic ash over Europe during the eruption of Eyjafjallajökull on Iceland, April-May 2010. Atmos Env 48:1-8, doi:10.1016/j.atmosenv.2011.03.054

Maeno F, Nakada S, Nagai M, Kozono T (2013) Ballistic ejecta and eruption condition of the vulcanian explosion of Shinmoedake volcano, Kyushu, Japan on 1 February, 2011. Earth Planets Space 65:609-621

Mastin LG (2008) A simple calculator of ballistic trajectories for blocks ejected during volcanic eruptions, version 1.4. US Geol Sur Open File Rep 01-45

Miura K (2008) Temporal-Color Coder., http://rsb.info.nih.gov/ij/macros/TemporalColor_Code.txt

Rietze M (2010) Iceland 6.5.2010-11.5.2010 Eyjafjallajökull Video Clips., http:// www.mrietze.com/iceland10-2.htm. Accessed 1 May 2013

Ripepe M, Bonadonna C, Folch A, Delle Donne D, Lacanna G, Marchetti E, Höskuldsson A (2013) Ash-plume dynamics and eruption source parameters by infrasound and thermal imagery: the 2010 Eyjafjallajökull eruption. Earth Planet Sci Lett 366:112-121, doi:10.1016/j.epsl.2013.02.005

Schneider CA, Rasband WS, Eliceiri KW (2012) NIH Image to ImageJ: 25 years of image analysis. Nat Methods 9:671-675

Sparks RSJ, Bursik MI, Carey SN, Gilbert JS, Glaze LS, Sigurdsson H, Woods AW (1997) Volcanic plumes. John Wiley \& Sons, Chichester

Suzuki YJ, Koyaguchi T (2013) 3D numerical simulation of volcanic eruption clouds during the 2011 Shinmoedake eruptions. Earth Planets Space 65:581-589

Taddeucci J, Scarlato P, Capponi A, Del Bello E, Cimarelli C, Palladino DM, Kueppers U (2012) High-speed imaging of Strombolian explosions: the ejection velocity of pyroclasts. Geophys Res Lett 39, L02301, do:10.1029/ 2011GL050404

Tsunematsu K, Chopard B, Falcone J-L, Bonadonna C (2014) A numerical model of ballistic transport with collisions in a volcanic setting. Comput Geosci 63:62-69, doi:10.1016/j.cageo.2013.10.016

Woodhouse MJ, Hogg AJ, Phillips JC, Sparks RSJ (2013) Interaction between volcanic plumes and wind during the 2010 Eyjafjallajökull eruption, Iceland. J Geophys Res 118:1-18, doi:10.1029/2012JB009592 